\section{A doença periodontal na comunidade negra dos Arturo's, Contagem, Minas Gerais, Brasil}

\author{
Periodontal disease in the Arturo's black \\ community in Contagem, Minas Gerais, Brazil
}

Takeshi Kato Segundo 1 Efigênia Ferreira e Ferreira 1 José Eustáquio da Costa ${ }^{2}$

\footnotetext{
1 Departamento de Odontologia Social e Preventiva, Faculdade de Odontologia, Universidade Federal de Minas Gerais, Belo Horizonte, Brasil.

2 Departamento de Clínica Patológica e Cirurgia Odontológica, Faculdade de Odontologia, Universidade Federal de Minas Gerais, Belo Horizonte, Brasil.

Correspondência Efigênia Ferreira e Ferreira Rua Rio Negro 1260/301, Belo Horizonte, $M G$ 30430-400, Brasil. efigenia@uai.com.br
}

\begin{abstract}
This was a cross-sectional study on periodontal disease in a black community in Brazil. The sample consisted of 104 individuals over 13 years of age ( 63 females and 41 males). All teeth were examined except the third molars. Six sites per tooth were analyzed in order to measure pocket depth and clinical loss of attachment. Four sites per tooth were used to measure bleeding on probing and presence of calculus. It was observed that bleeding on probing occurred in 97.9\% of the total of individuals with teeth, with probing depth $\geq 4 \mathrm{~mm}$ in $43.3 \%$ and clinical attachment loss in 63.5\%. Calculus was detected in $81.2 \%$ of the sample. In the study, individuals 46 to 60 years of age showed the highest mean clinical attachment loss, 8.3mm ( \pm 4.27$)$, and a probing depth of $4.6 \mathrm{~mm}( \pm 3.5)$. Prevalence rate for periodontal disease in this community was similar to rates elsewhere in Brazil and the world,i.e., 9.6\% for the severe form. Age, schooling, and tobacco use were identified as risk indicators statistically associated with periodontal disease.
\end{abstract}

Periodontal Disease; Prevalence; Risk Indicators

\section{Introdução}

A doença periodontal sofreu várias releituras durante as últimas décadas, em face dos avanços no campo da epidemiologia e mudanças dos enfoques conceituais e metodológicos. Dessa forma, obtiveram-se informações que estão contribuind o para a compreensão dessa infecção e os determinantes de sua extensão social e individual, como problema de saúde-doença bucal 1 .

Pode ser caracterizada, hoje, como um processo inflamatório causado por bactérias orais que acomete os tecidos gengival e ósseo. Sua gravidade está relacionada com má higiene bucal, presença de bactérias patogênicas, fumo e idade avançada. A doença causa a destruição dos tecidos em surtos aleatórios e em sítios específicos, podendo levar à perda do elemento se não controlada 2 .

Os sinais clínicos ou parâmetros clínicos indicadores de doença periodontal são características não pertinentes ao quadro fisiológico de saúde, os quais isolados, ou em associação com outros parâmetros, fornecem-nos dados para diagnosticar presença ou ausência de inflamação. São eles:

a) Sangramento gengival - o sangramento gengival é usado como índice de inflamação dos tecidos gengivais, tomando-se como princípio de que a sondagem leve e cuidadosa do sulco gengival não produz sangramento. A ausência de sangramento à sondagem sob uma 
força de 30 gramas indica uma estabilidade periodontal com um valor preditivo negativo de 98\%-99\%, sendo esse parâmetro clínico o mais seguro para controlar pacientes por períodos de tempo na prática diária 3,4 .

b) Cálculo supragengival - o cálculo dental é o fator retentivo de placa mais importante; no entanto, é um fator etiológico secundário na periodontite. Sua presença torna impossível a remoção adequada da placa e impede uma boa higienização por parte dos indivíduos 1,5.

c) Profundidade de sondagem (PS) - a PS é quantificada pela medida em milímetros da margem gengival ao fundo do sulco ou bolsa periodontal. Segundo Greenstein 6, estudos clínicos têm demonstrado que a PS não é um bom preditor de futura progressão da doença periodontal; entretanto, sítios com PS aumentada são de maior risco para progressão da doença do que sítios com menor PS. Além disso, o aumento da PS em um sítio aumenta o risco de progressão da doença ali.

d) Perda de inserção (PI) - a PI pode ser medida pela distância do limite amelo-cementário ao fundo do sulco ou bolsa periodontal. O monitoramento longitudinal das alterações ocorridas nas medidas do nível de inserção é um dos meios mais utilizados e confiáveis para avaliar progressão da doença periodontal, podendo ser considerado o padrão ouro 7 .

Os estudos epidemiológicos sobre a doença periodontal utilizam os indicadores já relacionados, sistematizados ou não em índices. A Organização Mundial de Saúde preconiza o uso do Índice Comunitário de Necessidades de Tratamento Periodontal (CPITN), modificado para Índice Periodontal Comunitário (IPC), em virtude do novo entendimento da patogênese da doença periodontal 8. Apesar desta adaptação, a crítica a este índice refere-se à substancial discrepância que pode ocorrer entre as avaliações que adotam análises parciais e aquelas que envolvem toda a dentição ${ }^{9}$.

É preciso destacar que existem críticas com relação à metodologia empregada em estudos. Irfan et al. 10 observaram que os estudos epidemiológicos carecem de consistência metodológica e padronização nos desenhos de estudo, prejudicando a comparação, o que é essencial para interpretação de risco e causalidade.

Alguns estudos são amplos, procurando medir a doença periodontal através de vários indicadores. Baelum et al. 11, por exemplo, avaliaram a saúde periodontal de 1.131 indivíduos com idade entre 15 a 65 anos em Machakos
District, Kênia. Foram observados mobilidade dentária, placa, cálculo, sangramento gengival, perda de inserção e profundidade de sondagem. A higiene oral encontrada foi deficiente, $\mathrm{e}$ $75 \%$ a $95 \%$ das superfícies examinadas possuíam placa. As superfícies com cálculo aumentavam consideravelmente com a idade: de $10 \%$ a $20 \%$ (15 a 24 anos) para $70 \%$ a $85 \%$ (55 a 65 anos). Independentemente da idade, a profundidade de sondagem $\geq 4 \mathrm{~mm}$ foi encontrada em menos de $20 \%$ da população estudada, e menos de $3 \%$ possuíam profundidade de sondagem $\geq 6 \mathrm{~mm}$.

No Brasil, o dado nacional existente foi obtido mediante um levantamento epidemiológico da saúde bucal 12, no qual foi possível dimensionar a prevalência da doença periodontal na população brasileira com mais de 15 anos de idade, residente em região urbana. Empregou-se o índice CPITN, em dez dentes-índice, com utilização do maior valor apurado por sextante. Foram consideradas a faixa etária (15 a 19,35 a 44 e 50 a 59 anos) e a renda familiar (até 2, 2-4 e 5 e mais salários mínimos). O quadro epidemiológico observado mostrou-se menos grave do que o esperado, fato explicado não pela melhor saúde, mas pelo alto número de desdentados (49,7\%, aos 50-59 anos). Quando esses dados foram analisados considerando-se a renda familiar, a necessidade cirúrgica observada em indivíduos com renda familiar de cinco salários mínimos ou mais foi de $0,4 \%$, $4,5 \%$ e $5,6 \%$ nas respectivas faixas etárias examinadas, e na faixa de renda de até dois salários mínimos, $1 \%$, 5,5\% e 8,8\% respectivamente.

O fator sócio-econômico tem sido considerado atualmente como de fundamental importância na determinação das doenças. Craig et al. 13, avaliando o risco da doença periodontal em asiáticos, africanos e espanhóis de Nova York, sugeriram que o fator sócio-econômico pode ser considerado como fator de risco para doença periodontal destrutiva mais forte do que a raça/etnia.

Poulton et al. 14 observaram a influência de uma infância em ambiente de nível sócio-econômico baixo e a saúde resultante na fase adulta, comparando os dados aos de um grupo com infância de alto nível sócio-econômico. A medida da saúde dental mostrou diferenças significativas entre os dois grupos, não só quanto à doença periodontal $(31,1 \%$ e $11,9 \%$, respectivamente), mas também quanto ao nível de cárie (32,2\% e 9,9\%, respectivamente) e à dependência do álcool $(21,5 \%$ r 12,1\%, respectivamente). 
A situação sócio-econômica determina também o acesso aos serviços, com graves conseqüências para a saúde, como é relatado por Harris 15 e Checchi et al. 16, que observaram estabilização ou agravamento do quadro diagnosticado inicialmente em pacientes que trataram e não trataram a doença periodontal, respectivamente.

Entende-se atualmente que a doença periodontal possui uma etiologia multifatorial, e os fatores causais estão em íntima relação com os indicadores de risco. Vários indicadores de risco potenciais para a doença periodontal têm sido analisados e confirmados pelos levantamentos epidemiológicos, tais como: sexo masculino 17 , origem negra ou filipina 18 , idade avançada 19, baixas condições sócio-econômicas ou educacionais 20, diabetes e fumo 21,22,23.

Em uma exploração mais profunda do indicador de risco raça negra, vários estudos relataram uma maior prevalência da doença periodontal em negros do que em brancos 2,11,18,24,25.

Beck et al. 18 estudaram a prevalência e indicadores de risco para a perda de inserção periodontal em idosos acima de 65 anos das raças branca e negra. Todos os dentes foram avaliados, concluindo-se que, em negros, $78 \%$ dos sítios avaliados tinham perda de inserção com média de $4 \mathrm{~mm}$, e, em brancos, $65 \%$ dos sítios apresentavam perda média de $3,1 \mathrm{~mm}$. No grupo de negros, foram considerados fatores de risco o uso de tabaco, a presença de Porphyromonas gingivalis maior que $2 \%$ e mais de três anos desde a última visita ao dentista. Para os brancos, essas mesmas características, à exceção da microbiota, também foram consideradas como fatores de risco.

Löe \& Brown 2 avaliaram estudantes com idade entre 14 e 17 anos, objetivando, através da verificação da perda de inserção (superfície mesial), classificar os casos de periodontite de início precoce, encontrada em $0,53 \%$ dos indivíduos. A raça negra apresentou maior risco para todas as formas de periodontite, e os homens negros possuíam 2,9 vezes mais chance de apresentar periodontite de início precoce do que as mulheres negras.

Brown et al. 24 avaliaram a perda de inserção após 18 meses em mais de mil idosos com idade superior a 65 anos. Os negros foram mais susceptíveis do que os brancos. Em 33\% de negros e $25 \%$ de brancos, houve perda de inserção em pelo menos dois sítios. No total, $24 \%$ de negros e $16 \%$ de brancos tiveram três ou mais sítios com perda de inserção $>3 \mathrm{~mm}$.

Drake et al. 25 avaliaram durante três anos a perda de elemento dentário entre dois grupos de idosos, um branco e outro negro. Observa- ram que $53 \%$ de negros e $29 \%$ de brancos perderam pelo menos um dente no período e que $13 \%$ de negros e $4 \%$ de brancos perderam todos os dentes remanescentes, mostrando que idosos negros possuem maior risco de perda dentária que idosos brancos. Para ambas as raças, bactérias orais e fator sócio-econômico aumentaram o risco de doença periodontal.

Esses trabalhos formam o marco conceitual desta pesquisa que tem como objetivo conhecer a prevalência e distribuição da doença periodontal na comunidade dos Arturo's, além de relacioná-la com os indicadores de risco aos quais estão expostos.

A comunidade em questão foi fundada em 1917 por um escravo alforriado, Artur Camilo, que juntamente com sua esposa, também exescrava, estabeleceram-se em uma chácara na região de Contagem, Minas Gerais, Brasil. Hoje cerca de 380 descendentes formam a Comunidade dos Arturo's, de muito prestígio no meio cultural mineiro em virtude da manutenção de suas tradições culturais africanas, seja na religião, como o Congado, seja nos dialetos usados entre eles. Através de um censo realizado, observou-se que a renda mensal na comunidade varia de um a três salários mínimos por residência.

\section{Metodologia}

Foi realizado um estudo seccional transversal, permitindo um retrato instantâneo da situação de saúde da população, com base na avaliação individual do estado de saúde periodontal de cada um dos membros do grupo, mediante exame clínico.

Como critério de inclusão no estudo, utilizou-se a idade acima de 13 anos, pois, teoricamente, é a idade em que todos os dentes permanentes já estão em oclusão, sem a presença de falsas bolsas acarretadas pelo processo de erupção dentária.

Através do cálculo amostral, verificou-se a necessidade de avaliar 73 indivíduos. Deste modo, por haver 115 indivíduos maiores de 13 anos, optou-se pelo recenseamento da população residente na chácara.

A avaliação diagnóstica da doença periodontal foi realizada a partir de um exame clínico em todos os dentes presentes com exceção dos terceiros molares, por causa da presença de falsas bolsas, tão comum no sítio distal dos mesmos e por serem dentes cuja presença na cavidade bucal é cada vez mais rara.

A opção de avaliar todos os dentes presentes, e não apenas os dentes-índice, deveu-se à 
quantidade de informações perdidas nos registros parciais, que subestimam a prevalência da doença. Um exame em toda dentição fornece os melhores meios de avaliar, de forma precisa, a prevalência da doença periodontal 5,26.

O exame clínico foi realizado dentro de uma igreja da própria localidade, utilizando-se EPI (equipamento de proteção individual) completo, gaze, quando necessário, sonda periodontal milimetrada tipo Willians e espelho bucal estéreis. Uma cadeira de madeira foi confeccionada para este fim. A iluminação foi feita com uma lanterna (tipo minerador - Micro Petzl) fixada na cabeça do examinador. Uma auxiliar treinada funcionou como anotadora.

Foram utilizadas, como indicadores de doença periodontal, a PS e a PI, ambas medidas com a sonda periodontal, e a presença de sangramento, medida 30 a 60 segundos após a sondagem. A opção de avaliar a presença de cálculo deveu-se à sua relação com o grau de higienização e à destruição periodontal.

Os critérios para cada indicador, baseados em Lindhe 5, foram:

a) Profundidade de sondagem (PS): foi realizada medindo-se a distância da margem gengival ao fundo do sulco ou bolsa gengival, incluindo seis sítios: disto-vestibular, vestibular, mésio-vestibular, disto-lingual, lingual e mésio-lingual;

b) Perda de inserção (PI): foi efetuada medindo a distância entre a junção cemento-esmalte e o fundo do sulco ou bolsa gengival, incluindo os sítios supracitados;

c) Sangramento à sondagem: fez-se a leitura do sangramento após a sondagem até o fundo do sulco ou bolsa gengival nas superfícies mesial, distal, vestibular e lingual;

d) Presença de cálculo: foi verificada por inspeção visual, nas superfícies supragengivais mesial, distal, vestibular e lingual.

Um questionário também foi respondido por cada indivíduo visando a coletar dados relacionados à exposição aos indicadores de risco, a saber: idade, gênero, diabetes, problemas cardiovasculares, tabagismo e tempo decorrido desde a última consulta ao dentista.

A análise dos dados do exame clínico e do questionário foi realizada utilizando-se o programa Epinfo 6.0. Os resultados foram expressos em porcentagem para as prevalências, medidas de tendência central (média) e dispersão (desvio padrão).

Com relação à profundidade de sondagem e perda de inserção considerou-se a faixa de 1 a $3 \mathrm{~mm}$ como saudável; 4 a $6 \mathrm{~mm}$, doença moderada; maior que $7 \mathrm{~mm}$, doença grave 27 , além do percentual de ocorrência e média. $\mathrm{Na}$ análise multivariada, os indivíduos que apresentaram doença moderada e grave foram reagrupados como "doentes".

Com relação ao sangramento gengival e à presença de cálculo, foram consideradas as médias e as porcentagens de acometimento na população estudada.

Para a análise estatística, os testes de significância utilizados foram Mann-Whitney, Kruskkal-Wallis; para a probabilidade de ocorrência, Odds Ratio 28.

\section{Resultados}

Foram examinados 104 indivíduos maiores de 13 anos de idade, do total de 115 residentes na chácara dos Arturo's, sendo 63 do gênero feminino e 41 do gênero masculino. Destes, oito eram edêntulos totais. De uma maneira geral, as condições periodontais apresentam-se nesta população conforme a descrição na Tabela1.

Para todas as outras análises, os edêntulos totais foram eliminados da amostra, que passou a perfazer um total de 96 indivíduos $(84 \%$ do total de residentes na chácara).

Considerando-se os pacientes dentados, a presença de cálculo foi detectada em $81,2 \%$ (78) dos indivíduos examinados. O sangramento à sondagem apresentou uma prevalência de 97,9\% (94 indivíduos).

Esses percentuais de sangramento gengival foram observados também quando se consideraram as variáveis gênero (100\% das mulheres e 94,6\% dos homens), escolaridade (96,9\% com até quarta série e $98,4 \%$ acima da quinta série), tabagismo (98,7\% dos não fumantes e 95,2\% dos fumantes), diabetes (97,8\% dos não diabéticos e $100 \%$ dos diabéticos) e alteração cardíaca $(98,8 \%$ dos indivíduos sem alteração e 93,8\% dos indivíduos com alteração). Em nenhum desses casos foi observada diferença estatisticamente significante.

A presença de cálculo foi detectada em $81,2 \%$ dos indivíduos, ocorrência alta se comparada aos dados encontrados por Gesser et al. $29,50,7 \%$ ou Tomita et al. $30,17 \%$ de ocorrência, ambos em população brasileira. Por ser um dificultador da higiene bucal, o cálculo pode ter contribuído para a prevalência de sangramento gengival (97,9\%), dado indicativo de gengivite. Em apenas dois indivíduos não se observou a ocorrência de sangramento à sondagem, prevalência muito alta, quando comparada à encontrada por Hornig et al. 27, em uma clínica militar: $37 \%$. No entanto, esses resultados são similares ao de Costa 1, que, em um estudo realizado em sua cidade, verificou a presença de san- 
Caracterização por faixa etária das variáveis: cálculo, sangramento à sondagem, pontos sangrantes e perda de inserção.

\begin{tabular}{|c|c|c|c|c|c|c|c|c|}
\hline \multirow[t]{2}{*}{ Faixa etária (anos) } & \multicolumn{2}{|c|}{$\begin{array}{l}\text { Superfícies } \\
\text { com cálculo }\end{array}$} & \multicolumn{2}{|c|}{$\begin{array}{l}\text { Superfícies com } \\
\text { sangramento }\end{array}$} & \multicolumn{2}{|c|}{ Pontos sangrantes } & \multicolumn{2}{|c|}{ Perda de inserção } \\
\hline & Média & $\mathrm{Dp}$ & Média & $\mathrm{Dp}$ & Média & $\mathrm{Dp}$ & Média & $\mathrm{Dp}$ \\
\hline $13-20$ & 6,23 & 11,52 & 24,08 & 19,94 & 3,26 & 0,82 & 3,75 & 1,88 \\
\hline $21-35$ & 20,18 & 24,16 & 37,90 & 25,71 & 4,33 & 1,42 & 4,97 & 1,89 \\
\hline $36-45$ & 24,15 & 22,75 & 26,21 & 20,00 & 3,84 & 1,89 & 5,52 & 3,23 \\
\hline $46-60$ & 18,16 & 20,31 & 25,33 & 19,80 & 4,66 & 3,50 & 8,33 & 4,27 \\
\hline Mais de 60 & 13,50 & 18,55 & 6,83 & 10,17 & 1,66 & 2,34 & 3,58 & 4,14 \\
\hline
\end{tabular}

gramento em $100 \%$ da amostra avaliada. Em outro estudo realizado no Brasil, Gesser et al. 29 observaram uma prevalência de $86 \%$.

O levantamento nacional 31 apresentou dados mais positivos para a população brasileira com renda similar (61\% de presença de sangramento e $48 \%$ de cálculo) e ainda melhor se considerada somente a Região Sudeste $(42 \%$ e $40 \%$, respectivamente). Deve-se lembrar que esta população, além de renda média de três salários mínimos, possui baixa escolaridade ( $32 \%$ até quarta série) e pouco acesso aos serviços de atendimento odontológico, sendo os dois últimos fatores agravantes para a saúde.

A perda de inserção variou de 1 a $13 \mathrm{~mm}$. Do total da amostra, em 61 indivíduos $(63,5 \%)$ foi detectada, em pelo menos um sítio, uma PI $\geq$ $4 \mathrm{~mm}$.

Em relação ao gênero, pôde-se verificar que $55,9 \%$ dos indivíduos do gênero feminino e $75,7 \%$ do gênero masculino possuíam PI $\geq$ $4 \mathrm{~mm}$, grupo em que foram observadas as maiores perdas (11 a 13mm), diferença esta não significante (Mann-Whitney, $\mathrm{p}>0,05$ ).

Considerando a escolaridade, a PI $\geq 4 \mathrm{~mm}$ foi observada em $84,4 \%$ dos indivíduos com até a quarta série e $53,2 \%$ dos indivíduos acima da quinta série; a análise estatística demonstrou que essa diferença foi estatisticamente significante (Mann-Whitney, $\mathrm{p}=0,003$ )

Quanto ao fato de o indivíduo ser ou não fumante, $57,3 \%$ dos não fumantes e $85,7 \%$ dos fumantes possuíam PI $\geq 4 \mathrm{~mm}$. A análise estatística mostrou que existe diferença estatisticamente significante entre os fumantes e os não fumantes (Mann-Whitney, $\mathrm{p}=0,018$ ).

Quando uma análise por faixa etária foi realizada (Tabela 1 ), verificou-se um aumento da PI com o aumento da idade. A perda média inicial de $3,7 \mathrm{~mm}$ (13 a 20 anos) progride para $4,97 \mathrm{~mm}$ ( 21 a 35 anos), 5,52mm (36 a 45 anos) e 8,3mm (46 a 60 anos). Em virtude da progressão da doença, que acarreta perdas dentárias, a média de PI diminui na faixa etária com mais de 60 anos, sendo de $3,6 \mathrm{~mm}$, mas, excetuandose esta faixa etária, a diferença foi confirmada estatisticamente (Kruskal-Wallis, p > 0,05), indicando existir uma relação entre a perda de inserção e a faixa etária.

Através do tratamento estatístico dos dados, não foi possível relacionar a perda de inserção com a última consulta ao dentista e com o fato de o indivíduo ser ou não diabético, ou possuir alguma alteração cardíaca.

A perda de inserção $\geq 4 \mathrm{~mm}$ em pelo menos um sítio foi detectada em $63,5 \%$. Em um estudo realizado no Sudão, Darout et al. 32 verificaram uma prevalência de PI $\geq 4 \mathrm{~mm}$ em $51 \%$ da amostra avaliada, resultado semelhante aos deste estudo.

A escolaridade e o tabagismo parecem ser fatores de risco para perda de inserção neste grupo (Mann-Whitney, $\mathrm{p}=0,003$ e $\mathrm{p}=0,018$, respectivamente). Locker \& Leake 22, Ismail et al. 33 e Elter el al. 34 relataram que a baixa escolaridade é um indicador de risco para doença periodontal. $\mathrm{O}$ tabagismo também é considerado fator de risco significativo. Stoltemberg et al. 35 concluíram que fumantes possuem cinco vezes mais chance de possuir PI $\geq 4 \mathrm{~mm}$, do que não fumantes, muito próximo do encontrado neste estudo, cuja odds ratio foi de 4,46.

A relação de aumento da PI com o aumento da idade é também observada nas pesquisas de Haffajee et al. 7, Hornig et al. 21, Locker \& Leake 22, Ismail et al. 33, Bragamian et al. 36, Grossi et al. 37, Mumghamba et al. 25 e confirmada neste estudo (Kruskal-Wallis, p > 0,05).

Com relação à profundidade de sondagem $\geq 4 \mathrm{~mm}$, a prevalência foi de $46,9 \%$. Considerando a classificação de Horning et al. 27, observouse entre os dentados um percentual de $36,4 \%$ 
de periodontite moderada ( $\mathrm{PS} \geq 4 \mathrm{e} \leq 6 \mathrm{~mm}$ ) e de $9,6 \%$ de periodontite avançada (PS $\geq 7 \mathrm{~mm}$ ). Em um estudo realizado no Kênia, Baelum et al. 11 observaram a PS $\geq 4 \mathrm{~mm}$ em menos de $20 \%$ da população estudada e menos de $3 \%$ possuíam $\mathrm{PS} \geq 6 \mathrm{~mm}$. Brown et al. 38 verificaram uma PS $\geq$ $7 \mathrm{~mm}$ em apenas $0,6 \%$ da amostra. No estudo epidemiológico do Ministério da Saúde 31, considerando a população de 15 a 59 anos, os indivíduos com $P S \geq 6 \mathrm{~mm}$ ocuparam o percentual de $4,1 \%$ (Brasil) e 5,4\% (Região Sudeste).

Ao analisar a PS média na faixa etária de 13 a 20 anos, $26,5 \%$ apresentaram PS $\geq 4$, prevalência muito alta, indicando uma possibilidade de ocorrência de periodontite juvenil; todavia, este dado não é conclusivo, visto que, neste estudo, foram analisadas apenas a PS e a PI, o que é insuficiente para diagnosticá-la. Estudos longitudinais da progressão da doença em adolescentes indicam que indivíduos com sinais de periodontite destrutiva em idade jovem estão predispostos à deterioração futura. Assim sendo, a deterioração parece ser mais pronunciada nos sítios inicialmente afetados, em pacientes diagnosticados com periodontite juvenil e oriundos das classes sócio-econômicas mais baixas 5,39. Desta maneira, podemos concluir que a alta prevalência da doença na faixa 13 a 20 anos segue um processo de destruição periodontal na faixa de 21 a 35 anos e atinge o maior grau de destruição na faixa de 46 a 60 anos, grupo etário em que foi encontrada a maior PS, com diferença estatisticamente significante (Mann-Whitney, $\mathrm{p}=0,031$ ). Por causa das perdas dentárias ocasionadas pela doença, a PS média diminui na faixa acima de 60 anos.

Em relação ao gênero, pôde-se verificar que $39,0 \%$ dos indivíduos do gênero feminino e $59,5 \%$ do gênero masculino possuíam PS $\geq 4 \mathrm{~mm}$.
Observando a escolaridade, 50,0\% dos indivíduos que estudaram até a quarta série e 45,3\% dos indivíduos acima da quinta série mostraram $P S \geq 4 \mathrm{~mm}$. A última consulta ao dentista foi realizada em até 24 meses por $46,7 \%$ dos examinados e acima de 24 meses por $53,3 \%$.

Dos 45 indivíduos com PS $\geq 4 \mathrm{~mm}, 13$ são fumantes e 32 não o são. Entre os fumantes, $61,9 \%$ possuem $\mathrm{PS} \geq 4 \mathrm{~mm}$.

Dos que relataram ter diabetes, $60 \%$ apresentaram PS $\geq 4 \mathrm{~mm}$, percentual maior do que entre os não diabéticos (46,2\%). Com relação à presença de alguma alteração cardíaca relatada, no grupo de indivíduos com alteração 50,0\% têm PS $\geq 4 \mathrm{~mm}$.

É importante ressaltar que nenhum dos fatores acima pesquisados foi estatisticamente relacionado com a profundidade de sondagem.

A fim de possibilitar uma visão geral dos indicadores de doença pesquisados e suas correlações com os indicadores de risco, foi elaborada a Tabela 2 .

A doença periodontal encontra-se dentro dos padrões mundiais em sua forma moderada e grave, ou seja entre $10 \%$ e $15 \%$ 5,40. Em termos de Brasil, o único estudo de prevalência que se tem é o levantamento epidemiológico do MS 31, no qual a prevalência é menor. Contudo, foi usado o CPITN, o que leva a uma perda de alguns dados, não permitindo uma comparação direta com trabalhos epidemiológicos utilizando dados de todos os dentes.

Sendo assim, os indicadores de risco considerados internamente ao grupo, a escolaridade, a faixa etária e o tabagismo, que apresentaram relação estatisticamente significante com os indicadores PI e PS, parecem confirmar a teoria de sua atuação como agravantes da doença periodontal.

Tabela 2

Caracterização da presença de correlação estatisticamente significante, valor de $p<0,05$.

\begin{tabular}{llccc}
\hline & Cálculo & Sangramento & Pontos de inserção & Pontos sangrantes \\
\hline Idade & $0,005^{*}$ & 0,112 & $0,000^{*}$ & $0,031^{*}$ \\
Gênero & 0,973 & 0,073 & 0,052 & 0,052 \\
Escolaridade & $0,038^{\star}$ & 0,618 & $0,003^{*}$ & 0,666 \\
Uso de tabaco & 0,123 & 0,333 & $0,017^{*}$ & 0,118 \\
Diabetes & 0,272 & 0,739 & 0,084 & 0,886 \\
Última consulta & 0,141 & 0,976 & 0,184 & 0,421 \\
Alteração cardíaca & 0,726 & 0,204 & 0,109 & 0,784 \\
\hline
\end{tabular}

* Presença de correlação 


\section{Conclusões}

Através deste estudo realizado na comunidade negra dos Arturo's, pode-se concluir que:

- A prevalência da doença periodontal está dentro dos padrões do Brasil e do mundo, apresentando uma prevalência de $36,4 \%$ na forma moderada e $9,6 \%$ na forma grave.

- A hipótese da maior prevalência nesta população da raça negra não foi confirmada.

- Quanto aos fatores de risco pesquisados - a escolaridade, o uso do tabaco e a faixa etária eles mostraram relação com a PI, e somente a faixa etária mostrou relação com a PS.

Resumo

Trata-se de um estudo seccional transversal em uma comunidade da raça negra. A amostra foi constituída por 104 indivíduos acima de 13 anos de idade, sendo 63 mulheres e 41 homens. Foram examinados todos os dentes presentes, com exceção dos terceiros molares. Cada dente foi sondado em seis sítios para registrar a profundidade de sondagem e a perda de inserção e em quatro sítios para registrar sangramento após sondagem e presença de cálculo. Observou-se que, do total dos dentados, o sangramento à sondagem foi detectado em 97,9\%, a profundidade de sondagem (PS) $\geq$ $4 \mathrm{~mm}$ foi observada em 43,3\%, a perda de inserção (PI) $\geq 4 \mathrm{~mm}$ verificou-se em $63,5 \%$ e em $81,2 \%$ observou-se a presença de cálculo. De 46 a 60 anos, identificou-se uma PI de 8,3mm ( $\pm 4,27)$, a maior média, e também PS de 4,6mm ( $\pm 3,5)$. A doença acometeu $9,6 \%$ dos participantes em sua forma grave ( $P S \geq 7$ ), valor dentro dos padrões conhecidos no Brasil e no mundo, não havendo um diferencial por ser um povo negro. Faixa etária, escolaridade e uso de tabaco foram indicadores de risco relacionados com a doença.

Doença Periodontal; Prevalência; Indicadores de Risco

\section{Colaboradores}

Todos os autores participaram de todas as etapas de elaboração do artigo.

\section{Referências}

1. Costa FO. Doença periodontal de início precoce em crianças, adolescentes e adultos jovens: um estudo longitudinal da incidência, severidade e extensão [Tese de Doutorado] Belo Horizonte: Faculdade de Odontologia, Universidade Federal de Minas Gerais; 2000.

2. Löe H, Brown JL. Early onset periodontitis in the United States of America. J Periodontol 1991; 62:608-16.

3. Claffey N, Nylund K, Kiger R, Garrett S, Egelberg J. Diagnostic predictability of scores of plaque, bleeding, suppuration and pocket dephts for probing attachment loss: 3 ? years of observation following initial therapy. J Clin Periodontol 1990; 17:108-14.

4. Lang NP, Adler R, Joss A, Nyman S. Absence of bleeding on probing. An indictor of periodontal stability. J Clin Periodontol 1990; 17:714-21.

5. Lindhe JAN. Tratado de periodontia clínica e implantologia oral. 3a Ed. Rio de Janeiro: Guanabara Koogan; 1999.

6. Greenstein G. Contemporary interpretation of probing depth assessments; Diagnostic and therapeutic implications. A literature review. J Periodontol 1997; 68:1194-205.

7. Haffajee AD, Socransky SS. Attachment level changes in destrutive periodontal diseases. J Clin Periodontol 1986; 13 Suppl:461-72.

8. Organização Mundial da Saúde. Levantamento epidemiológico básico de saúde bucal: manual de instrução. 3a Ed. São Paulo: Santos; 1999. 
9. Baelum-Beck JD, Koch GG. Characteristics of older adults experiencing periodontal attachment loss as gingival recession or probing depth. J Periodontal Res 1994; 29:290-8.

10. Irfan UM, Dawson DV, Bissada NF. Epidemiology of periodontal disease: a review and clinical perspectives. J Int Acad Periodontol 2001; 3:14-21.

11. Baelum V, Fejerkov O, Manji F. Periodontal disease in adult Kenians. J Clin Periodontol 1988; 15:445-52.

12. Ministério da Saúde. Levantamento epidemiológico em saúde bucal: Brasil, zona urbana, 1986. Brasília: Centro de Documentação do Ministério da Saúde; 1988.

13. Craig RG, Yip JK, Mijares DQ, Boylan RJ, Haffajee AD, Socransky SS. Destructive periodontal diseases in minority populations. Dent Clin North Am 2003; 47:103-14.

14. Poulton R, Caspi A, Milne BJ, Thomson WM, Taylor A, Sears MR, et al. Association between children's experience of socioeconomic disadvantage and adult health: a life-course. Lancet 2002; 23: 1619-20.

15. Harris RJ. Untreated periodontal disease: a follow-up on 30 cases. J Periodontol 2003; 74:672-8.

16. Checchi L, Montevecchi M, Gatto MR, Trombelli L. Retrospective study of tooth loss in 92 treated periodontal patients. J Clin Periodontol 2002; 29 : 651-6.

17. Calsina G, Ramón JM, Echeverría JJ. Effects of smoking on periodontal tissues. J Clin Periodontol 2002; 29:771-6.

18. Beck JD, Koch GG, Rozier RG, Tudor GE. Prevalence and risk indicators for periodontal attachment loss in a population of older communitydwelling blacks and whites. J Periodontol 1990; 61:521-8.

19. Ogawa H, Yoshihara A, Hirotomi T, Ando Y, Miyazaki H. Risk factors for periodontal disease progression among elderly people. J Clin Periodontol 2002; 29:592-7.

20. Skaleric U, Kovac-Kavcic M. Some risk factors for the progression of periodontal disease. J Int Acad Periodontol 2000; 2:19-23.

21. Horning MG, Hatch CL, Cohen ME. Risk indicators for periodontitis in a military treatment population. J Periodontol 1992; 63:297-302.

22. Locher D, Leake JL. Risk indicators and risk markers for periodontal disease experience in older adults living independently in Ontario, Canada. J Dent Res 1993; 72:9-17.

23. Mumghamba EG, Markkanen HA, Honkala E. Risck factors for periodontal disesse in Ilala, Tanzânia. J Clin Periodontol 1995; 22:347-54.

24. Brown FL, Beck JD, Rozier RG. Incidence of attachment loss in community-dewlling older adults. J Periodontol 1994; 65:316-23.

25. Drake CW, Hunt RJ, Koch GG. Three-year toth loss among black and white older adults in North Carolina. J Dent Res 1995; 74:675-80.
26. Eaton KA, Duffy S, Griffiths GS, Gilthorpe MS, Johnson NW. The influence of partial and fullmouth recordings on estimates of prevalence and extent of lifetime cumulative attachment loss: a study in a population of young male militay recruits. J Periodontol 2001; 72:140-5.

27. Horning MG, Hatch CL, Cohen ME. The prevalence of periodontitis in a military treatment population. J Am Dent Assoc 1990; 121:616-22.

28. Soares JF, Siqueira AL. Introdução à estatística médica. Belo Horizonte: Departamento de Estatística, Universidade Federal de Minas Gerais; 2001.

29. Gesser HC, Peres MA, Marcenes W. Condições gengivais e periodontais associadas a fatores sócio-econômicos. Rev Saúde Pública 2001; 35:28993.

30. Tomita NE, Cordeiro R, Mendonça JS, Senger V. Lopes ES. Saúde bucal dos trabalhadores de uma indústria alimentícia do Centro-Oeste paulista. Revista da Faculdade Odontologia de Bauru 1999; 7:67-71.

31. Ministério da Saúde. Levantamento epidemiológico em saúde bucal: Brasil, zona urbana, 1986. Brasília: Centro de Documentação do Ministério da Saúde; 1988.

32. Darout IA, Albandar JM, Skaug N. Periodontal status of adult Sudaneses habitual uses of miswak chewing sticks or toothbrushes. Acta Odontol Scand 2000; 58:25-30.

33. Ismail AI, Eklund AS, Striffer DF, Szpunar SM. The prevalence of advanced loss of periodontal attachment in two New Mexico populations. J Periodontal Res 1987; 22:119-24.

34. Elter JR, Beck JD, Slade GD, Offenbacher S. Etiologic models for incident periodontal attachment loss in older adults. J Clin Periodontol 1999; 26: 113-23.

35. Stoltemberg JL, Osborn JB, Pihlstrom BL, Herzberg MC, Aeppli DM, Wolff LF, et al. Association between cigarette smoking, bacterial pathogens, and periodontal status. J Periodontol 1993; 64:1225-30.

36. Bragamian RA, Farghaly MM, Lopatin D, Sowers M, Syed AS, Pomerville JL. Periodontal disease in Amish population. J Clin Periodontol 1993;20:26972.

37. Grossi SG, Genco RJ, Machtei EE, Ho AW, Koch G, Dunford R, et al. Assessment of risk for periodontal disease. I. Risk indicators for attachment loss. J Periodontol 1994; 65:260-7.

38. Brown JL, Oliver RC, Löe H. Evaluating periodontal status of US employed adults. J Am Dent Assoc 1990; 121:226-32.

39. Albandar JM, Baghadady VS, Ghose LJ. Periodontal disease progression in teenagers with no preventive dental care provisions. J Clin Periodontol 1991; 18:300-4.

40. Papapanou PN. Periodontal diseases: epidemiology: Ann Periodontol 1996; 1:1-36.

Recebido em 21/Mai/2003

Versão final reapresentada em 13/Out/2003

Aprovado em 28/Out/2003 